\title{
Influence of the Spectral Quality of Light on Daytime Alertness Levels in Humans
}

\author{
Kamila Łaszewska', Agnieszka Goroncy ${ }^{2}$, Piotr Weber ${ }^{3}$, Tadeusz Pracki ${ }^{4}$, and \\ Małgorzata Tafil-Klawe ${ }^{4}$
}

\begin{abstract}
'Department of Psychology, Faculty of Humanities, Nicolaus Copernicus University, Fosa Staromiejska 1a, 87-100 Toruń, Poland ${ }^{2}$ Department of Mathematical Statistics and Data Analysis, Faculty of Mathematics and Computer Science, Nicolaus Copernicus University, Chopina 12/18, 87-100 Toruń, Poland

${ }^{3}$ Atomic and Optical Physics Division, Department of Atomic, Molecular and Optical Physics, Faculty of Applied Physics and Mathematics, Gdańsk University of Technology, Narutowicza 11/12, 80- 233 Gdańsk, Poland

${ }^{4}$ Department of Human Physiology, Nicolaus Copernicus University Ludwik Rydygier Collegium Medicum in Bydgoszcz, Karłowicza 24, 85-092 Bydgoszcz, Poland
\end{abstract}

ABSTRACT

Exposure to light is very important for human health. However, the characteristics of the light stimulus and the appropriate timing of such exposure are essential. Studies that have used monochromatic light exposure have shown no systematic patterns for the effects of blue light compared to longer wavelengths. Previous studies have shown that red light exposure increases objective and subjective measures of alertness at night without suppressing nocturnal melatonin or inducing circadian disruption. The present study investigated whether noon time exposure to red light would increase both objective and subjective measures of alertness such as those measured by EEG, cognitive-behavioral performance, and subjective sleepiness. The three lighting conditions were as follows: dim light ( $<0.01$ lux at cornea), blue light $(465 \mathrm{~nm}, 72 \mu \mathrm{W} / \mathrm{cm} 2)$, and red light (625 $\mathrm{nm}, 18 \mu \mathrm{W} / \mathrm{cm} 2)$, both at 40 lux. The results of the EEG data showed an increase in theta power over time in dim light only. In red light, alpha power showed a decrease over time. The impact of red light was observed in the performance measures: The only significant effect was a deterioration in the continuous performance test after red light exposure. Subjective measures of alertness were not affected by light in either condition, in contrast to darkness, when subjects reported greater sleepiness than before. None of the changes in objective measures of alertness induced by red light exposure translated into subjective sleepiness at noon. Thus, we concluded that behavioral effects of light at noon are very limited at best.

light,

daytime,

alertness,

performance,

EEG

\section{INTRODUCTION}

Light not only provides visual information, but also exerts a nonvisual response that influences many processes, both physiological and behavioral. Bright light is an important human Zeitgeber, causing time-dependent cycling changes in hormone secretion, sleep-wake regulation, thermoregulation, performance, and alertness (Dijk \& Archer, 2009). Alertness is promoted during the day, whereas sleepiness is promoted at night. Unfortunately, these changes are not always consistent with social or psychological demands.

The above-mentioned conflicts between natural human activity and social demands occur both at night and in the daytime. The problem of regulating the use of light radiation according to its detriments and benefits during nighttime and in the late afternoon has already been widely discussed (Ben-Shlomo \& Kyriacou, 2010; Crowley, Lee, Tseng, Fogg, \& Eastman, 2003; Czeisler, 2009; Eastman, Stewart, Mahoney, Liu, \& Fogg, 1994; Fonken et al., 2009; Martin \& Eastman, 1997; Penev, Kolker, Zee, \& Turek, 1998; Revell \& Eastman, 2005; Schernhammer \& Schulmeister, 2004; Smith \& Eastman, 2008). However, there is a gap Corresponding author: Kamila Łaszewska, Department of Psychology, Faculty of Humanities, Nicolaus Copernicus University, Fosa Staromiejska 1a, 87-100 Toruń, Poland. E-mail: kamila.laszewska@gmail.com 
in the knowledge concerning the nonvisual alerting response (NVAR) to light radiation during the day. There are some peak times during the bright time of the $24 \mathrm{~h}$ day when homeostatic sleep pressure is the highest: the early hours of the morning and the middle of the afternoon (Vanlaar, 2008). The latter occurs particularly between 2 PM and 4 PM (The Royal Society for the Prevention of Accidents [ROSPA], 2011), and is called the post-lunch dip (Monk, 2005). It may be the cause of accidents in many areas of industry and transportation (Maycock, 1996), as "falling asleep at the wheel" is a major cause of road accidents in Great Britain, accounting for up to $20 \%$ of serious accidents on motorways and monotonous roads (Reyner \& Horne, 2000; ROSPA, 2008, 2011). Thus, there is a need to propose solutions as to how to avoid daytime sleepiness. We hypothesize that the alerting features of light might be used. However, we first need to know more about the features of the NVAR during the day especially (a) how NVAR sensitivity changes throughout the daytime, (b) on which wavelengths it is most sensitive, (c) whether we are conscious of these changes, and (d) how it impacts our physiology and cognitive functions.

This NVAR is associated with several processes: the suppression of nocturnal melatonin (Lockley, Evans, Scheer, Brainard, Czeisler, \& Aeschbach, 2006; Lockley \& Gooley, 2006), cortisol secretion (Lockley et al., 2006; Scheer \& Buijs, 1999) changes in pupil size (Brainard et al., 2001; Dijk \& Archer., 2009), core temperature and heart rate (Cajochen et al., 2005), performance capability (Lockley et al., 2006), mood (Mills, Tomkins, \& Schlangen, 2007; Vandewalle et al., 2010), and alertness levels (Cajochen et al., 2011; Cajochen, Zeitzer, Czeisler, \& Dijk, 2000; Lockley et al., 2006).

Correlates of alertness might be subjective (high subjective alertness ratings, low subjective fatigue ratings) and objective (behavior and brain activity). Alertness is associated with levels of some endogenous hormones-that is, melatonin and core body temperature (Figueiro, Bierman, Plitnick, \& Rea, 2009). In terms of cognitive-behavioral performance, alertness is also used to describe a state of vigilance or sustained attention in which the person is able to achieve and maintain a certain level of cognitive performance while executing a given task (Oken, Salinsky, \& Elsas, 2006). In addition to results on performance tests, reliable neurophysiological measurements of human alertness include electroencephalographic (EEG) measurements, with low power densities in the theta $(4-8 \mathrm{~Hz}$, Cajochen et al., 2005) and alpha frequency range (8-12 Hz, Figueiro et al., 2009), electrooculographic (EOG) slow rolling eye movements, and eye blink rate (Cajochen et al., 2005).

Pineal melatonin is selectively secreted during the night (Arendt, Bojkowski, Franey, Wright, \& Marks, 1985) and under conditions of darkness in both diurnal and nocturnal species (Arendt, Deacon, English, Hampton, \& Morgan, 1995). The rate of melatonin secretion is acutely attenuated by nocturnal light (Lewy, Wehr, Goodwin, Newsome, \& Markey, 1980) and the circadian rhythm is chronically regulated by the light-dark cycle (Czeisler \& Gooley, 2007). Since the peak spectral sensitivity of acute melatonin suppression is approximately $450 \mathrm{~nm}$ (Rea, Figueiro, Bullough, \& Bierman, 2005), shortwavelength light (seen as blue, $\mathrm{BL}$ ) is maximally effective at suppress- ing melatonin levels (Brainard et al., 2001). In controlled conditions, melatonin is considered to be the best available noninvasive marker of the internal clock in humans (Klerman et al., 2002). Therefore, in most studies to date, the NVAR effects of light have been associated with its ability to suppress melatonin, thereby implicating circadian system participation in this process (Cajochen et al., 2005; Lockley, Brainard, \& Czeisler, 2003; Lockley \& Gooley, 2006).

However, according to the most recent findings, acute melatonin suppression is not needed for light to have an effect on measures of alertness at night. Namely, it has been demonstrated that nighttime stimulation with long-wavelength light (RL, seen as red) produces an alerting response similar to the one observed in bioelectrical brain activity (increased beta and reduced alpha power) at 40 lux of BL, although only BL significantly suppressed melatonin levels (Figueiro et al., 2009; Plitnick, Figueiro, Wood, \& Rea, 2010). These results are consistent with the most recent findings presenting the nonvisual alerting effects of light during the daytime when melatonin levels are hardly detectable (Łaszewska et. al., 2017; Sahin \& Figueiro, 2013; Sahin, Wood, Plitnick, \& Figueiro, 2014). In the afternoon, exposure to RL radiation has been shown to significantly decrease power density in the theta, alpha theta (Sahin \& Figueiro, 2013), and alpha ranges (Sahin \& Figueiro, 2013; Sahin et al., 2014), whereas at noon, it has been shown to decrease synchronization in the upper theta, lower alpha, and upper alpha EEG activity spectrum (Laszewska et. al, 2017). This leads to the suggestion that, at night, light-induced alertness can arise from neural pathways other than the circadian one.

The NVAR depends upon the characteristics of light exposure (intensity, spectrum, duration). Because the findings concerning RL (Figueiro et al., 2009; Łaszewska et al., 2017; Sahin \& Figueiro, 2013; Sahin et al., 2014) are puzzling and are contrary to the knowledge about the influence of BL presented in previous studies, the current study has been designed to further confirm these results and to investigate the issue at the brightest part of the $24 \mathrm{~h}$ day-at noon (Klejna et al., 2014).

The influence of RL might help answer the question as to whether the NVAR is induced by a nonvisual system in a way that differs from the circadian one since this radiation does not affect melatonin levels. We assume, in this case, that this will be observed during the daytime, the time when circadian influence associated with melatonin secretion supporting the alerting response to light is hardly detectable.

The aim of the present study is to compare the influence of BL (465 $\mathrm{nm}$ ) with RL (625 nm) on objective and subjective measures of alertness during the day, so that it will be possible to further develop the next part of the lighting scheme. We hypothesize that both BL and RL, in comparison to the dark condition (DC), will enhance the alertness level as observed in (a) the decrease of the power density of theta (4.0$8.0 \mathrm{~Hz}$ ) and alpha waves (8.0-12.0 Hz) in EEG data; (b) improveme time and the number of correct reactions in cognitive tests; and (c) decrease of subjective sleepiness in the Karolinska Sleepiness Scale (KSS, Åkerstedt \& Gillberg, 1990). Since the effects of light exposure on performance are inconsistent (Souman, Tinga, Te Pas, Van Ee, \& Vlaskamp, 2017), we decided to examine other cognitive functions. We assumed that light is a robust countermeasure for subjective sleepi- 
ness and for performance reduction, lessening the growing noontime sleepiness in humans. Therefore, we investigated whether the alertness induced by a very low light level was subjectively perceived during the day.

\section{METHODS}

\section{Participants and Screening Procedures}

Nineteen healthy males (19-35 years; $M d n=22$; lower quartile $=20$, upper quartile $=33$ ) were recruited for the study. Only males were enrolled in the study because hormonal changes in females would require additional, very complicated controls in the study protocol (Cahill, 2006). The study took place in the Department of Human Physiology of The Ludwik Rydygier Collegium Medicum in Bydgoszcz. The protocol was approved by the university's Committee of Bioethics. A written consent form was obtained from the participants before enrollment in the study. Participants were informed about the study and about its safety and were ensured that they could withdraw from the study at any time without giving the reason. Subjects enrolled in the study voluntarily, encouraged by advertisement at universities and web postings, and through word of mouth.

Participants who completed the study were extensively screened by a trained psychologist. They were diagnosed as healthy and did not report any physical or mental conditions. Seasonal affective disorder (SAD) was diagnosed via psychological interview and in accordance with the Polish version of the Automated Personal Inventory of Depression and SAD (AutoPIDS-S, Terman \& Williams, 2015; enrollees had a score of 0 ) Mood disorders were evaluated as well, also on the basis of a psychological interview which, translated into responses on the Beck Depression Scale (Beck, Steer, Carbin, 1988) resulted in less than 11 points; $M=4.10 \pm 1.97$. They were diagnosed as neither extremely late nor early types (according to their answers on the Polish version of the Morningness-Eveningness Questionnaire, MEQ; Horne \& Östberg, 1976), free from sleep disorders and experiencing good sleep quality, which was assessed by psychological examination, with participants scoring no higher than 5 points on the Polish version of the Pittsburgh Sleep Quality Index (Buysse, Reynolds, Monk, Berman \& Kupfer, 1989), $M=2.0 \pm 0.88$, and no higher than 9 points on the Epworth Sleepiness Scale, (Johns, 1991), $M=3.32 \pm 1.63$. The enrolled participants denied taking pharmaceutics or medications and declared to be nonsmokers. They had a normal body mass index (BMI, Hall \& Cole, 2006), $M=23.6 \pm 3.33 ; \min =18.62 ; \max =31.02$, and had no color blindness (as measured by the Ishihara color blindness test, Lakowski, 1969). The participants had not traveled to more than one time zone within the 2 months prior to the study and had not worked night shifts within 6 months prior to the experiment.

Participants were asked to maintain a fixed $8 \mathrm{~h}$ regular sleep schedule for seven nights preceding the experiment and to wake up no later than $4 \mathrm{hrs}$ before visiting the laboratory, which was verified by sleep logs and actigraphy (Actiwatch AW4) one week before the study. This data was assessed by the quality, not quantity of the record. The participants were instructed to refrain from drinking alcohol and caffeine beginning from the afternoon before the experimental session. None of the individuals were heavy coffee drinkers. The participants were also asked to take care of the quality of their night sleep.

\section{Lightning Conditions}

The conditions of the experiment enable the observation of acute changes during exposure to the light stimulus. Three conditions were delivered to each subject: two experimental lighting conditions and the DC ( $<0.01$ lux at cornea). This study compared the influence of $\mathrm{BL}$ and RL. We were interested in whether both wavelengths were effective in modulating brain activity, being the objective measure of alertness, during the day. The study was designed in such a way that sleepiness could occur from its beginning till the end. It was hypothesized that over time, compared to the DC, participants exposed to RL and $\mathrm{BL}$ as dim as 40 lux would become less tired. We assumed that if light triggers a nonvisual response via pathways other than melatonin suppression, the RL would trigger a nonvisual response similar to BL. Monochromatic light with short- and long-wavelength was delivered to the corneas of individual subjects by arrays of light-emitting diodes (LEDs; ICove, Color Kinetics). They were placed in a $0.6 \mathrm{~m} \times 0.6 \mathrm{~m}$ $\times 0.6 \mathrm{~m}$ light box (as shown in Figure 1), and to be outside the subject's direct view, they were located behind the front box apertures. Moreover, in order to achieve a nonglaring and uniform distribution of light within the box, it was painted white. The spectral emissions of the short-wavelength LEDs peak at $465 \mathrm{~nm}$ (LXHL-MD1D Luxeon lamp) and for long-wavelength, at $625 \mathrm{~nm}$ (HPR8C-48KBD Luxeon lamp) both with full width at half maximum (FWHM) of $25 \mathrm{~nm}$. Although it has been proposed that lux might be a less appropriate unit for estimating the influence of light on the nonimage forming effects of retinal light exposure, we equated the stimuli according to the orthodox photopic luminous efficiency function (lux; Lucas et al., 2014; Vandewalle, Maquet \& Dijk, 2009). However, as we wanted to extend the results of Figueiro (2009) we used lux as they did. Another important reason for doing so was that lux has sensitivity at long wavelengths. Finally, since light in rooms is equated according to lux, it would be more useful for the preliminary assessments of the influence of light stimuli on humans in their environment and for the planning of further studies. However, we have taken into consideration the discussion that short-wavelength light equated in lux is a stronger stimulus than the long-wavelength: 72 $\mu \mathrm{w} / \mathrm{cm} 2-16.9 \times 1012$ photon/s cm 2 at 40 lux for BL and $18 \mu \mathrm{w} / \mathrm{cm} 2-$ $56 \times 1012$ photon/s cm 2 at 40 lux for RL. Thus, compared to previous research, a very low light level was used in our study. An additional reason for prescribing only 40 lux was to examine the level similar to that emitted by mobile phones and to find the lowest level at which the response changes. Prior to the experiment, the spectral radiances of both lighting conditions were measured with a calibrated spectroradiometer and were used to calibrate the gigahertz illuminance readings. In order to achieve the prescribed light levels before each experiment, light levels were be adjusted with an electronic dimmer. 


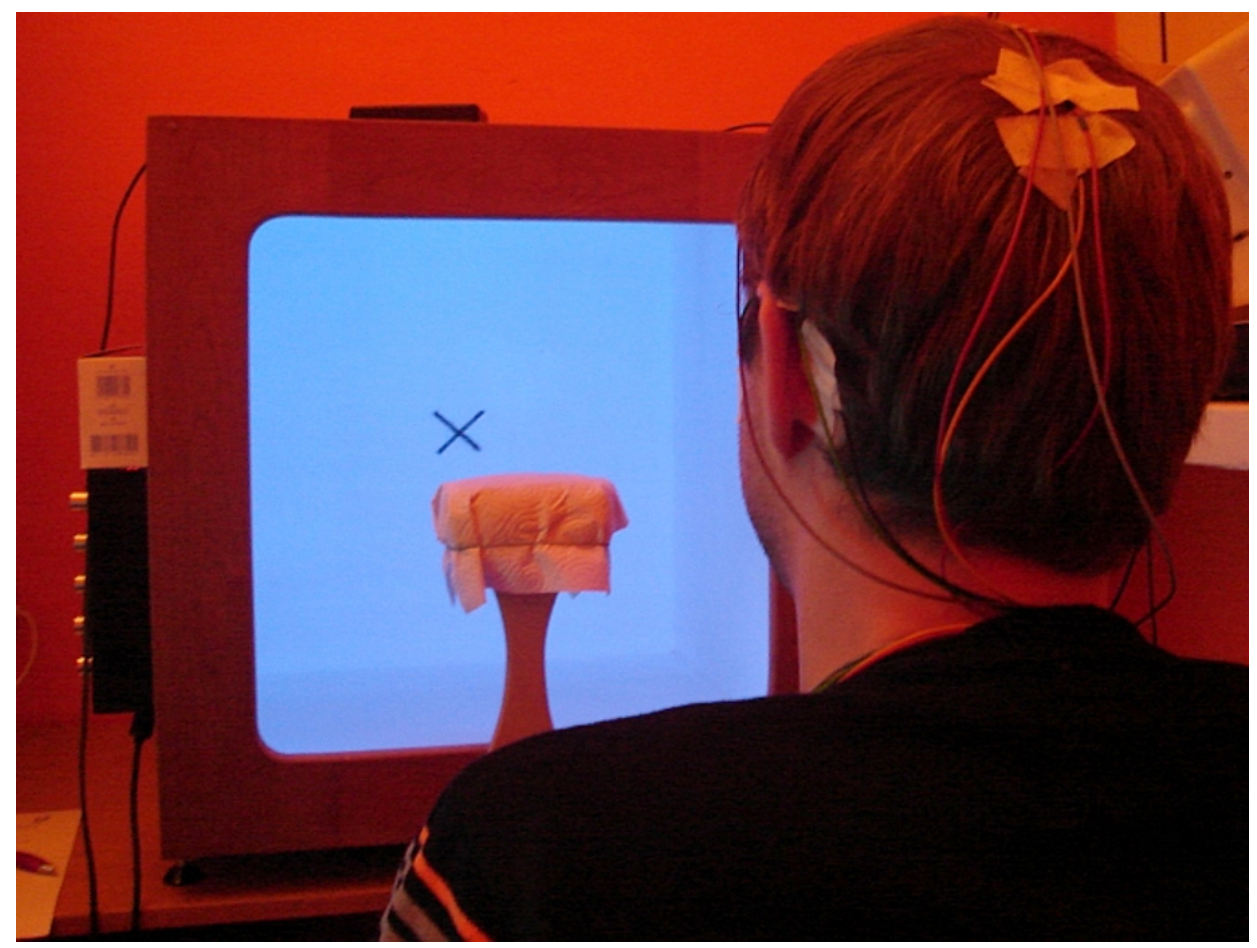

FIGURE 1.

Participant sitting at the light box before the experiment in a comfortable position.

\section{Objective Measures of Alertness}

\section{ELECTROENCEPHALOGRAM}

For EEG recordings and analyses, an EEG DigiTrack system (Elmiko Medical) was used. Electrodes were placed on the participants' scalps according to the International 10-20 system (Zyss, Hesse, \& Zięba, 2009). Digitally, the following four channels were recorded from the scalp: $\mathrm{Fz}, \mathrm{Cz}, \mathrm{Pz}$, and $\mathrm{Oz}$. Two additional electrodes serving as virtual reference electrodes were attached to the right and to the left earlobes. To provide aid, two derivations of the electroocularogram (EOG) for the left eye, EOG1 (FP1), and for the right eye, EOG2 (FP2), according to two reference electrodes $(\mathrm{A} 1+\mathrm{A} 2)$, were placed on the left and right earlobes. The purpose was to monitor artifacts caused by eyeblinks, which may produce voltage above $100 \mu \mathrm{V}$ and which may spread within the scalp, resulting in changes in the EEG. Eye movements may cause wavelengths similar to slow wavelengths, which are present only during wakefulness in subjects with mental or psychiatric disorders. Optical artifacts were cut with a Butterworth filter. The signal recorded from derivations was probed with a frequency of $120 \mathrm{~Hz}$ and a resolution of 12 bits.

\section{PERFORMANCE}

A battery of three psychomotor cognitive tests was used to measure performance: Simple Reaction Time (SRT), N-back (1-back version) and a Continuous Performance Test II (CPT II), all of which are described below. One week before the first session, each participant visited the lab in order to become familiar with and practice the tests. During such training, the participants practiced the tests at least twice (they had to receive a minimum of $80 \%$ correct answers). It was assumed that due to the training, the stress concerning the performance of a new task impairing cognitive functions would be avoided

Simple Reaction Time. This tool assesses alertness levels and attention as it corresponds with correct responses to rare stimuli. The SRT measures simple reaction time through the delivery of 25 known visual stimuli (in the version used, a cross was presented) to a known location on a computer screen. As soon as the stimulus was presented on the screen, the participant had to press the spacebar on the press pad. The outcome measures for SRT cover latency (response speed): the interval between the presentation of the stimulus and the appearance of an appropriate voluntary response in the subject (Jain, Bansal, Kumar, \& Singh, 2015).

$\mathbf{N}$-back, 1-back version. The N-back task is a frequently used continuous measure of working memory and working memory capacity in cognitive neuroscience research. It measures human psychomotor skills and visual-spatial working memory. In the current study, it was used to assess the participants' capacity to actively maintain and regulate a limited amount of task-relevant information (Baddeley \& Logie, 1999). The task consisted of 27 numbers visually presented in intervals lasting $1.8 \mathrm{~s}$. The length of the presentation of each stimulus was 0.2 s. The target response was to remember the numbers and to press the button on the press pad with the number that appeared previously: one item back on the computer screen. A response before the appearance of the next stimulus constituted an error. Reaction time in ms and the percentage of correct responses were assessed (Jansma, Ramsey, Coppola, \& Kahn, 2000). 
Continuous Performance Test II. The CPT is the most popular tool to obtain quantitative information regarding an individual's ability to sustain attention, cognitive efficiency, and impulsivity. In the current study, the more complex form of the CPT was used: the X-type CPT, namely, the CPT II.

The standard protocol of the CPT II uses a short practice exercise (lasting about $70 \mathrm{~s}$ ) prior to the administration of the full test to ensure that the respondent fully understands the task. In the main test applied in the current study, the letters were presented visually on a computer screen, one at a time, at a fixed rate of $920 \mathrm{~ms}$ between presentations. The subject was to respond by pressing a button (marked to be recognized in the DC) whenever the letter $X$ appeared and to inhibit responding when any other letter appeared. The CPT II respondents were also instructed to press another button (marked to be recognized in the DC) only if the $X$ was immediately preceded by the letter $A$. The display time was 50-75 ms. Targets constituted only $10-20 \%$ of all letters presented in one trail. The letter order varied between presentations. In assessing the results of the CPT II, the both commission errors (made when responses were given to nontargets) and omission errors (number of targets not responded to) were included.

The cognitive tests were presented on a computer screen, which emitted light. Since we wanted to avoid additional lights sources, we used special foil to block the emitted lights. For short-wavelength light, the Roscolux \#72 Azure Blue light filter was used and for long-wavelength-the Roscolux \#21 Golden Amber. In order to assess whether the different foils constituted a stimulus, we conducted an additional experiment to compare their influence on cognitive functions and the EEG results. Since in the results of this study we did not observe any statistically important differences, we concluded that neither foil interfered with our observations. We decided to use both foils during the cognitive tests. Depending on the experiment, one of the foils was carefully pasted on the computer screen before the subject arrived at the lab.

\section{Subjective Measures of Alertness/ Sleepiness}

For reports of instantaneous sleepiness (across the day and night) the KSS is often used (Akerstedt \& Gillberg, 1990). The KSS was originally developed to constitute a scale of subjective sleepiness and was validated against objective measures: both alpha and theta EEG activity as well as slow eye movement EOG activity (Åkerstedt \& Gillberg, 1990). The participants' current level of sleepiness was evaluated using a 9-point scale, where 1 corresponded to very alert, 3-rather alert, 5 - neither alert nor sleepy, 7-sleepy, but no difficulty remaining awake, and 9-very sleepy, fighting sleep, an effort to remain awake (Åkerstedt \& Gillberg., 1990). The participants filled out the KSS twice: before and after the EEG and EOG recordings (Kaida et al., 2006).

\section{Procedure}

The study was conducted between February 2012 and October 2012. One week before the experiment, each participant was familiarized with the procedure and the laboratory. Because only one light box and EEG machine was available, only one participant was examined at a given time. The research was scheduled each day at the same time, which minimized the effect of the time of day. The participants were asked to come to the laboratory at 11:00 AM to get instructions and be fitted with the scalp electrodes for EEG recordings. The session started at 12:00 PM, the time when melatonin is secreted at very low levels. This time of day was chosen because it is the time when humans are exposed to a large amount of light. Each participant took part in both experimental conditions and the DC, but only one at a time. The sessions were spaced 7 days apart. Due to participation in both the experimental and the control conditions, the measured subjects' psychophysiological parameters were unified. The session order was counterbalanced for each individual so that procedure familiarization would not influence the experiments results. The same number of sessions were on the same order place. All three sessions were conducted in the same conditions and schedules. The procedure was carefully controlled so as to accurately measure the response to the light stimuli. Because the procedure was complicated, we decided to avoid additional reactions that might emerge from using pupil dilators or the procedure of pupil measurement. For the sessions with light exposure, lights inside the box were energized until a steady level was achieved about $30 \mathrm{~min}$ before the experiment. After the fitting subjects with scalp electrodes for EEG recordings, they were asked to sit in a dimmed room isolated from other light sources, where the light exposure took place. The computer screen was placed near the light box so that they could sit comfortably in one place and start the light exposure just after the cognitive tests. Both the experimental sessions (with $465 \mathrm{~nm}$ and 625 $\mathrm{nm}$ ) and the DC session lasted 43 minutes and started with 15 minutes of sitting in dim light conditions $(<0.01$ lux) called the dark adaptation (Berson, Dunn, \& Takao, 2002; Wong, Dunn, \& Berson, 2005). During this inactive, dark period, the participants were kept in front of the light box with nonenergized LEDs, remained quiet, and were not allowed to perform any activity. At the beginning of every session, just before proper light exposure, the first performance and subjective sleepiness assessment took place. According to the requirement of the standard protocol of the CPT II, the administrator remained present while the test took place. Thus, the conditions remained constant: The administrator was the same person who behaved the same way in every session. He was not engaged in any contact with the subjects and his only task was to turn the computer on and off. In order to maintain the condition constancy, at the preparation period the subjects were instructed in a detailed way about the protocol of the study. Just before the cognitive tests, the computer screen was turned on by the experimenter. The participants who completed the cognitive tests filled out the KSS and scored their subjective level of sleepiness at that time. Just afterwards, the computer was turned off. After that time, in each session, the first EEG data collection took place. This part consisted of five active and five inactive phases when EEG and EOG activity were recorded. During both the experimental and controlled sessions, conditions were the same. During the active phase lasting $3 \mathrm{~min}$, the subjects remained seated and were asked to place their chin on a rest mounted near the front of the box to avoid head movements. They 
were instructed not to move their heads and bodies, to keep their eyes open and to focus on a black cross painted on the back wall of the light box, to avoid eye movements, and to refrain as much as possible from blinking, which in turn, could cause artefacts in the EEG data. To avoid any changes in EEG activity, they were asked to be relaxed and not to engage in any intellectual activity like problem solving. At the end, just after the light exposure, the second performance assessment with the use of the same cognitive tools was performed. The inactive phase was introduced so that the subjects could rest from the active phase, which required concentration and a forced position. The inactive part, on the other hand, lasted 5 minutes. In this part of the experiment, the subjects were instructed to remove their chins from the chinrest and sit comfortably. They could blink, but were not allowed to close their eyes for an extended period of time. The EEG and EOG recordings from this part was not analyzed. The active and inactive phases alternated and the changes were signaled by the sound of a bell. The total time of the active phase was set for 15 minutes, which means that the time of exposure in both experimental sessions (with BL and RL) lasted 15 minutes. Thus, the light session was a $40 \mathrm{~min}$ intermittent bright light exposure containing $15 \mathrm{~min}$ of bright light. The control conditions were conducted according to the same schedule with one exception: instead of being exposed to light, participants remained in the DC. In the active phase, when the cross in the light box was almost impossible to see, the subjects were instructed to fixate on a specific marked point approximately one meter away inside the light box. Figure 2 summarizes the experimental protocol.

\section{Data Analysis}

A spectral analysis was carried out on EEG signals from the participants during light exposure. Each signal from one electrode and one period of light exposure (21,600 points taking with $120 \mathrm{~Hz}$ sampling frequency) was divided into smaller fragments $\left(2^{8}=256\right.$ points $)$. We analyzed three frequency ranges inside the $4-30 \mathrm{~Hz}$ frequency domain. Then, we used a bandpass filter with a passband from 4 to $30 \mathrm{~Hz}$. This filter was used to filter each 28 point fragment. Next, we calculated the power density spectrums of each fragment according to the following formula:

$P_{k}=\left(\frac{\left|f_{k}\right|}{N}\right)^{2}, \quad k=1,2, \ldots$ where $\left|f_{k}\right|$ is modulus of kth value of discrete Fourier transform. The spectral power densities obtained $\left(P_{\mathrm{k}}\right)$ were grouped according to the following ranges in the frequency domain: $4 \mathrm{~Hz}-8 \mathrm{~Hz}$ (theta); $8 \mathrm{~Hz}$ $12 \mathrm{~Hz}$ (alpha); $13 \mathrm{~Hz}-30 \mathrm{~Hz}$ (beta). For each spectrum range, the area under the graph was counted according to the trapezoidal method. Taking into account all spectrums of fragments belonging to one active phase, we calculated the median power in each range in the frequency domain. These values were the objects of statistical consideration.

\section{RESULTS}

First, the exploratory data analysis was performed with use of the $\mathrm{R}$ statistical environment (version 3.1.2). Eventually, due to the normality violation, data was logarithmically transformed. These actions improved the quality of data such that the parametric analysis of variance (ANOVA) could be carried out. The ANOVAs and post hoc tests (pairwise multiple comparisons with the Bonferroni correction) were conducted using IBM SPSS Statistics (v23). In all cases the sphericity of data was examined via Mauchly's tests, however the details are not ad-

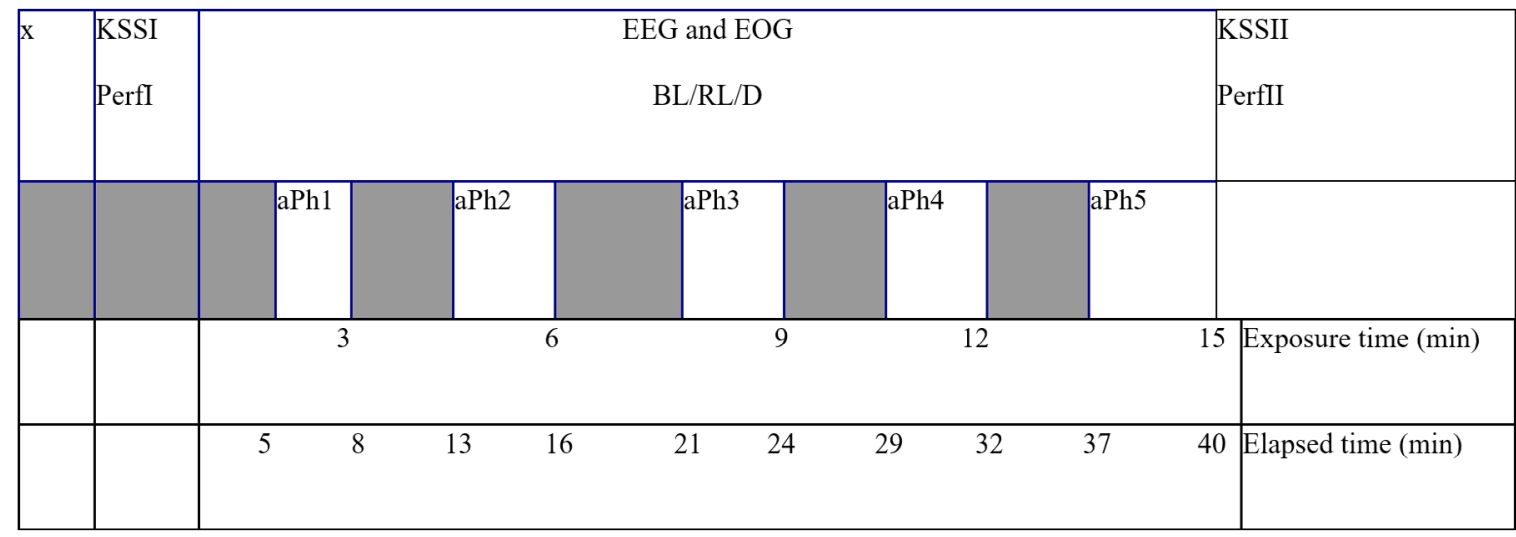

|FIGURE 2.

The study protocol for all three conditions. The bar with $x$ denotes the preparation period, where the participants remained seated in only dim light conditions. It lasted 15 min (dark adaptation). KSS I, Perf I denotes the first data collection which was placed just after the preparation period. Afterwards, one of the three conditions took place: blue light (BL) exposure, red light (RL) exposure, or the control condition (darkness, D). During every condition, EEG and EOG data were recorded. Squared bars denote inactive phases; bars marked $a P h 1$ to $a P h 5$ denote active phases. Exposure times are shown in minutes and indicate the total time of exposure, whereas elapsed time, also in minutes, denotes total time including inactive phases. Only the EEG and EOG recordings from the active phases were. KSS II, Perf II denotes the second data collection point just after the fifth active phase after the end of the EEG and EOG recordings. 
duced here. If the sphericity of some main effects or interactions could not be assumed, we corrected the F-tests' results using GreenhouseGeisser corrected degrees of freedom. Nevertheless, the original degrees of freedom are reported here. All statistical test results were considered significant if their respective significance values did not exceed .05. Tables presented in this section report only significant results.

\section{Power Density in Theta Frequencies of the Electroencephalogram}

The power density of the EEG theta frequencies was examined in order to find the dependencies between various experiment conditions.

We began with the three factorial repeated-measures ANOVA with a $3 \times 4 \times 5($ Light [BL, RL, DC] $\times$ Derivation $[\mathrm{Fz}, \mathrm{Cz}, \mathrm{Pz}, \mathrm{Oz}] \times$ Measurement Phase $[1,2,3,4,5])$ design design and the log-trans-

\section{TABLE 1.}

The Three-Factorial ANOVA Results for Theta Frequencies

\begin{tabular}{ccccc}
\hline Source & SSQ & df & $F$ & $p$ \\
\hline Light & 19.891 & 2 & 6.942 & .003 \\
Derivation & 18.278 & 3 & 16.233 & $<.001$ \\
Phase & 30.741 & 4 & 9.831 & $<.001$ \\
Light $\times$ Phase & 40.255 & 8 & 11.02 & $<.001$ \\
$\begin{array}{c}\text { Derivation } \times \text { Phase } \\
\text { Light } \times \text { Derivation }\end{array}$ & 9.366 & 12 & 9.534 & $<.001$ \\
$\times$ Phase & 6.146 & 24 & 2.908 & .004 \\
\hline
\end{tabular}

Note. SSQ = Type II sum of squares. formed EEG power spectral density as the dependent variable. The results are presented in Table 1 .

One can see that the interaction between all three effects proved to be significant, which implies that the EEG theta power density differs depending on the light conditions, derivation, and phase of the experiment. Post-hoc comparisons revealed that significant differences are only noticeable in the DC, in all derivations, mostly between the first three and the last two phases of the experiment (see Table 2). Respective means and error bars representing $\pm 1 \mathrm{SE}$ for the log-transformed data can be found in Figure 3. One can see that the means of EEG theta power in DC are significantly higher in the last two phases compared to the previous ones.

First, since we observed that the theta EEG power density differences in the last two phases seemed to behave quite differently from the previous ones, we wanted to explore these data to see whether the differences were significant. Respective means and error bars representing \pm 1 SE for the log-transformed data can be found in Figure 3 . Two 2-way repeated-measures ANOVAs with the following design, $3 \times$ 4 (Light [BL, RL, DC] $\times$ Derivation [Fz, Cz, Pz, Oz]), were conducted in the 4th and 5th phase of the experiment, respectively, for the same input data as in the three-way ANOVA.

Tests for within-subjects effects in the 4th phase of the experiment proved only the significance of the light effect, $\mathrm{SSQ}=29.052 ; \mathrm{ME}=$ 14.526; $F(2,36)=23.958 ; p<.001$, while the derivation effect and the interaction between light and the derivation effects turned out to be insignificant. Further post-hoc analyses revealed significant differ-

\section{TABLE 2.}

Significant Test Results of Pairwise Comparisons With the Bonferroni Correction for the Theta EEG Frequencies

\begin{tabular}{|c|c|c|c|c|c|c|c|}
\hline Derivation & Phase & Phase & $p$ & Derivation & Phase & Phase & $p$ \\
\hline \multirow[t]{7}{*}{$\mathrm{Fz}$} & 1 & 4 & .004 & \multirow[t]{7}{*}{$\mathrm{Cz}$} & \multirow[t]{3}{*}{1} & 2 & .029 \\
\hline & & 5 & .001 & & & 4 & $<.001$ \\
\hline & 2 & 4 & .023 & & & 5 & $<.001$ \\
\hline & 3 & 4 & $<.001$ & & \multirow[t]{2}{*}{2} & 4 & .008 \\
\hline & & \multirow[t]{3}{*}{5} & \multirow[t]{3}{*}{$<.001$} & & & 5 & .033 \\
\hline & & & & & 3 & 4 & .002 \\
\hline & & & & & & 5 & .001 \\
\hline \multirow[t]{6}{*}{$\mathrm{Pz}$} & 1 & 4 & .001 & \multirow[t]{6}{*}{$\mathrm{Oz}$} & \multirow[t]{2}{*}{1} & 3 & .027 \\
\hline & & 5 & $<.001$ & & & 5 & .045 \\
\hline & \multirow[t]{2}{*}{2} & 4 & .001 & & \multirow[t]{2}{*}{2} & 4 & .004 \\
\hline & & 5 & .001 & & & 5 & .005 \\
\hline & \multirow[t]{2}{*}{3} & 4 & $<.001$ & & \multirow[t]{2}{*}{3} & 4 & .003 \\
\hline & & 5 & $<.001$ & & & 5 & .001 \\
\hline
\end{tabular}


ences between $\mathrm{BL}$ and the $\mathrm{DC}(p<.001)$ and between $\mathrm{RL}$ and the $\mathrm{DC}$ $(p<.005)$ regardless of derivation, where the EEG theta power density was significantly higher in the DC than for BL. This is presented in Figure 3.

Analogous results were obtained for the 5th phase of the experiment. The significant main effects of the light and, additionally, of the derivation, were also discovered, as shown in Table 3. In particular, the post-hoc tests showed significant differences between BL and the DC $(p=.001)$ as well as between RL and the DC $(p=.003)$, which is illustrated in Figure 4, where the EEG theta power density is also significantly higher in the DC than in other light conditions. Regarding the main effect of derivation, significant differences were revealed between the $\mathrm{Oz}$ derivation, where the EEG theta power density was higher, and all other derivations (Fz: $p=.017$; Cz: $p=.041$; Pz: $p=.032)$, which is presented in Figure 5.

Another analysis concerned particular derivations: $\mathrm{Cz}$ and $\mathrm{Oz}$. Two-factorial repeated-measures ANOVAs were carried out to discover the differences between light and phases in these setups. In Table 4, we present significant results regarding the effect of phase and the interaction between the light and phase in the $\mathrm{Cz}$ derivation. In both the $\mathrm{Cz}$ and $\mathrm{Oz}$ derivations, the interaction of both effects was the subject of our interest, and thus, post-hoc comparisons were performed. They showed that significant differences occurred only in the DC, mainly between the first three phases (with lower values of theta frequencies) and the latter two (with higher values of theta frequencies), as presented in Table 2 (see Figure 6).
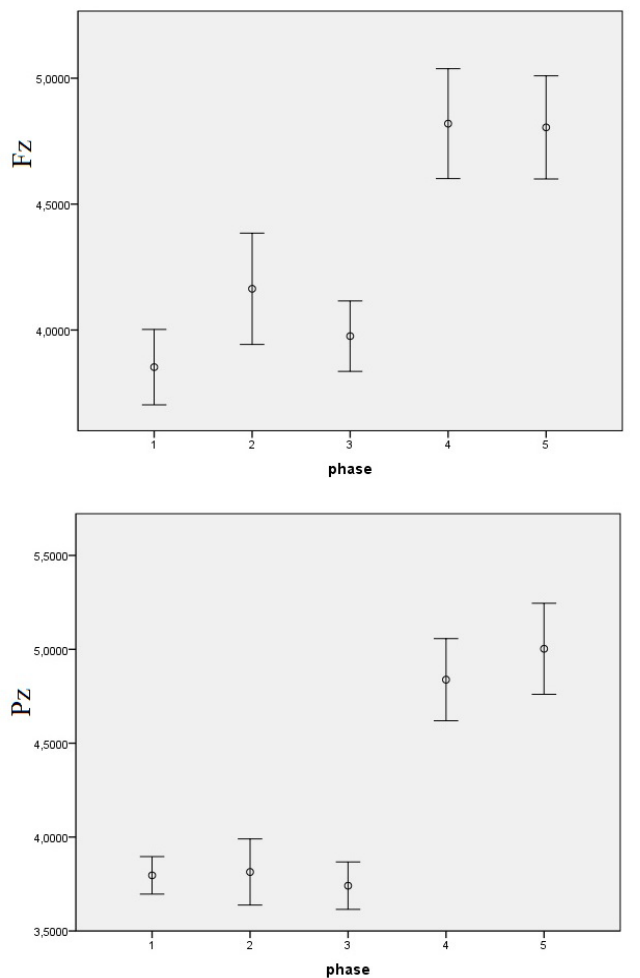

\section{TABLE 3.}

Two-Way Repeated-Measures ANOVA Results for Theta EEG Power Density in the 5 th Phase of the Experiment

\begin{tabular}{ccccc}
\hline Source & SSQ & df & $F$ & $p$ \\
\hline Light & 29.891 & 2 & 15.105 & $<.001$ \\
Derivation & 2.133 & 3 & 6.524 & .001 \\
\hline
\end{tabular}

Note. SSQ = Type II sum of squares.

- TABLE 4.

Two-Way Repeated-Measures ANOVA Results for Theta EEG Power Density in the Cz Derivation

\begin{tabular}{ccccc}
\hline Source & SSQ & df & $F$ & $p$ \\
\hline Phase & 3.526 & 4 & 4.725 & .005 \\
Light $\times$ Phase & 12.237 & 8 & 13.432 & $<.001$ \\
\hline
\end{tabular}

Note. SSQ = Type II sum of squares.

\section{TABLE 5.}

Two-way Repeated-Measures ANOVA Results for Theta EEG Power Density in the Oz Derivation

\begin{tabular}{ccccc}
\hline Source & SSQ & df & $F$ & $p$ \\
\hline Light & 8.77 & 2 & 7.634 & .002 \\
Phase & 6.174 & 4 & 6.357 & $<.001$ \\
Light $\times$ Phase & 12.365 & 8 & 8.243 & $<.001$ \\
\hline
\end{tabular}

Note. SSQ $=$ Type II sum of squares.
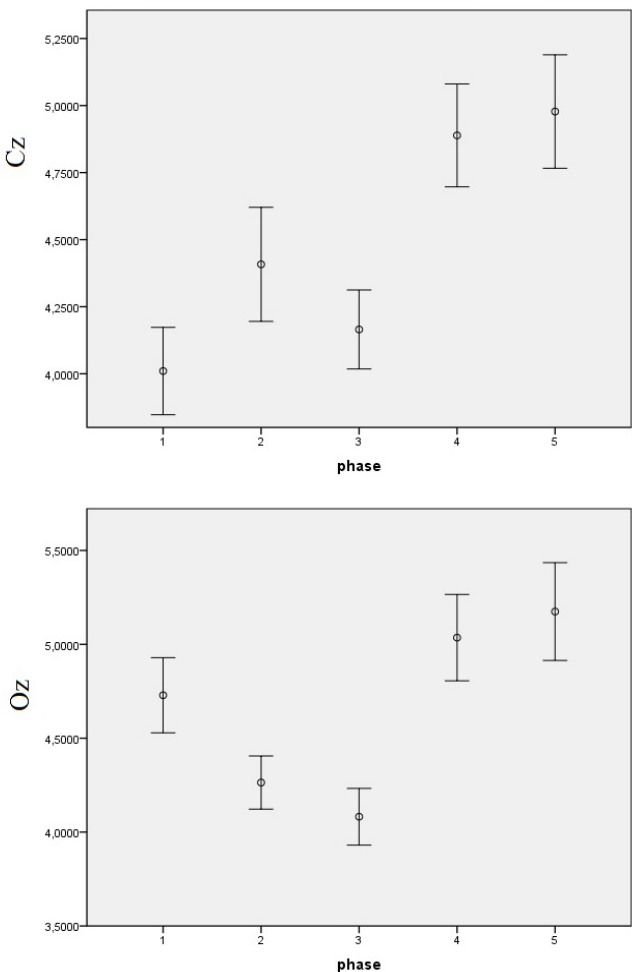

\section{FIGURE 3.}

Means of log-transformed theta EEG power density in various derivations ( $F z, C z, P z, O z)$, phases and light conditions with error bars representing $\pm 1 \mathrm{SE}$. 

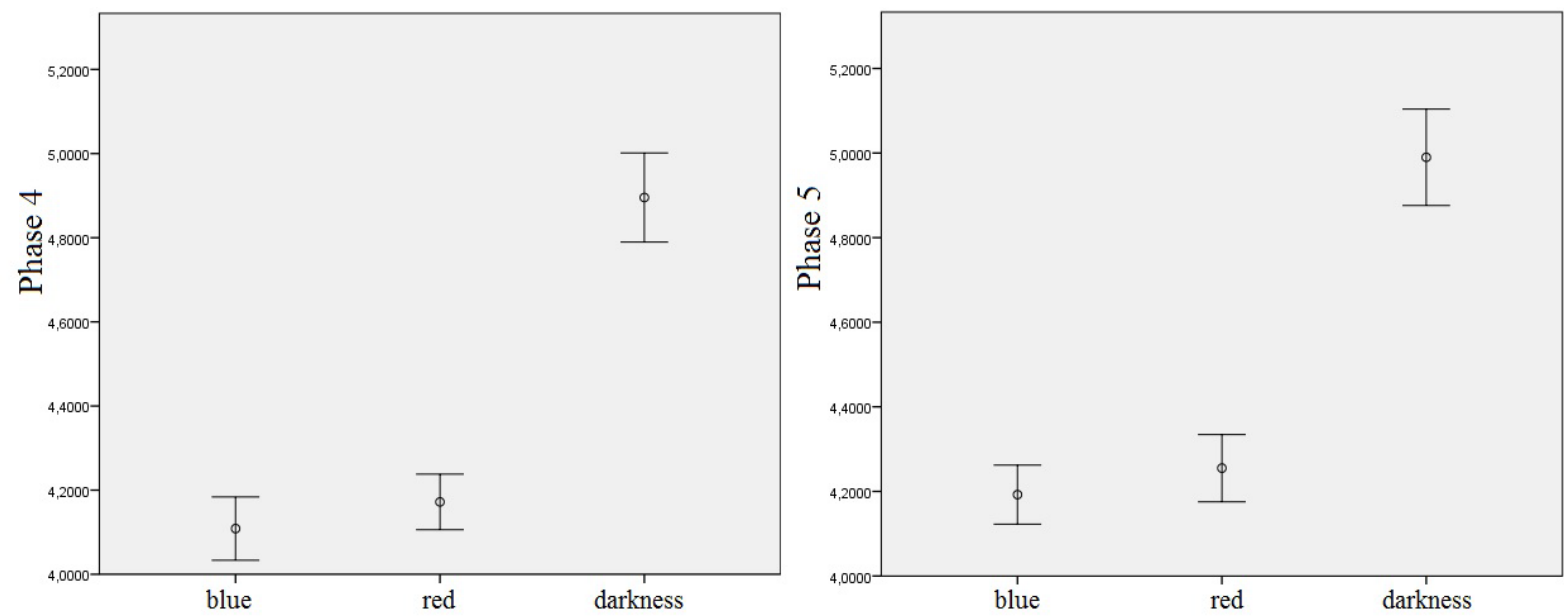

FIGURE 4.

Means of log-transformed theta EEG power density in the 4th and 5th phase of the experiment and different light conditions with error bars representing $+/-1 \mathrm{SE}$, regardless of the derivation.

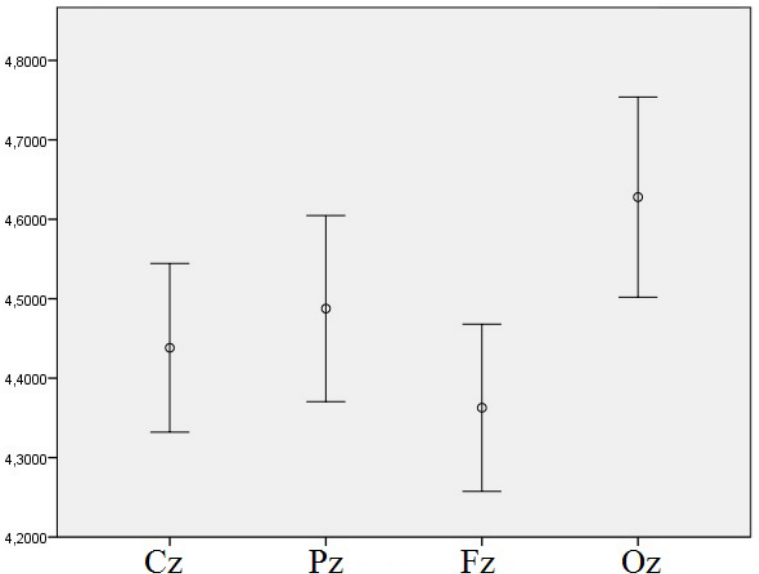

FIGURE 5.

Means of log-transformed theta EEG power density in the 5 th phase of the experiment and different derivations with error bars representing $\pm 1 \mathrm{SE}$, regardless of the light conditions.

\section{Power Density in Alpha Frequencies of the Electroencephalogram}

Another objective of the research was to analyze EEG power density in alpha frequencies. We started with the three-factorial repeated-measures ANOVA exactly in the same schema as for theta frequencies. The respective results of these tests are presented in Table 6 As seen above, the main effect of derivation and two of the two-way interactions were significant. Hence, the decision was made to interpret the latter interactions using post-hoc comparisons. First, as for the interaction between light and phase, significant differences occured in RL between the first and the fourth phase only, where the decrease of the alpha frequencies was noticed, and in the DC, between the third and last two phases of the experiment, when the alpha EEG power density was the highest. The corresponding significance values are presented in Table 7 . The
TABLE 6.

The Three-Factorial ANOVA Results for the Alpha Frequencies

\begin{tabular}{ccccc}
\hline Source & SSQ & df & $F$ & $p$ \\
\hline Derivation & 4.464 & 3 & 4.887 & .004 \\
Light $\times$ Phase & 18.324 & 8 & 4.362 & .001 \\
Derivation $\times$ Phase & 9.959 & 12 & 8.371 & .001 \\
\hline
\end{tabular}

Note. SSQ = Type II sum of squares.

\section{TABLE 7.}

The Three-Factorial ANOVA Results for the Alpha Frequencies

\begin{tabular}{cccc}
\hline Light condition & Phase & Phase & $p$ \\
\hline Red light & 1 & 4 & .029 \\
Darkness & 3 & 4 & .016 \\
& & 5 & .002 \\
\hline
\end{tabular}

respective means of the log-transformed alpha EEG power densities are illustrated in Figure 6.

Following the pairwise comparisons for the next two-way interaction of derivation and phase, we concluded that significant differences occur only in the Fz derivation, as shown in Table 8 and Figure 7 below. One can see that the alpha frequencies are significantly higher in the first phase than in the second one, and significantly lower in Phases 2 and 3 than in the last two phases, regardless of the light conditions.

We also performed two-factorial repeated-measures ANOVAs in the $\mathrm{Cz}$ and $\mathrm{Oz}$ derivations with the $3 \times 5$ (Light [BL, RL, DC] $\times$ Measurement Phase $[1,2,3,4,5])$ factor design and the log-transformed EEG power spectral density in the alpha frequencies as the dependent variable.

The results in the $\mathrm{Cz}$ derivation implied a significant difference in the alpha EEG power densities depending on the light and the measurement phase of the experiment, $F(8,144)=4.073, p=.004$. Post-hoc tests showed that significant differences occured only for RL, between Phases 1 and $4(p=.013)$, and between Phases 2 and $4(p=0.042)$, which is presented in Figure 8. In both cases the decrease of alpha frequencies is noticeable. 

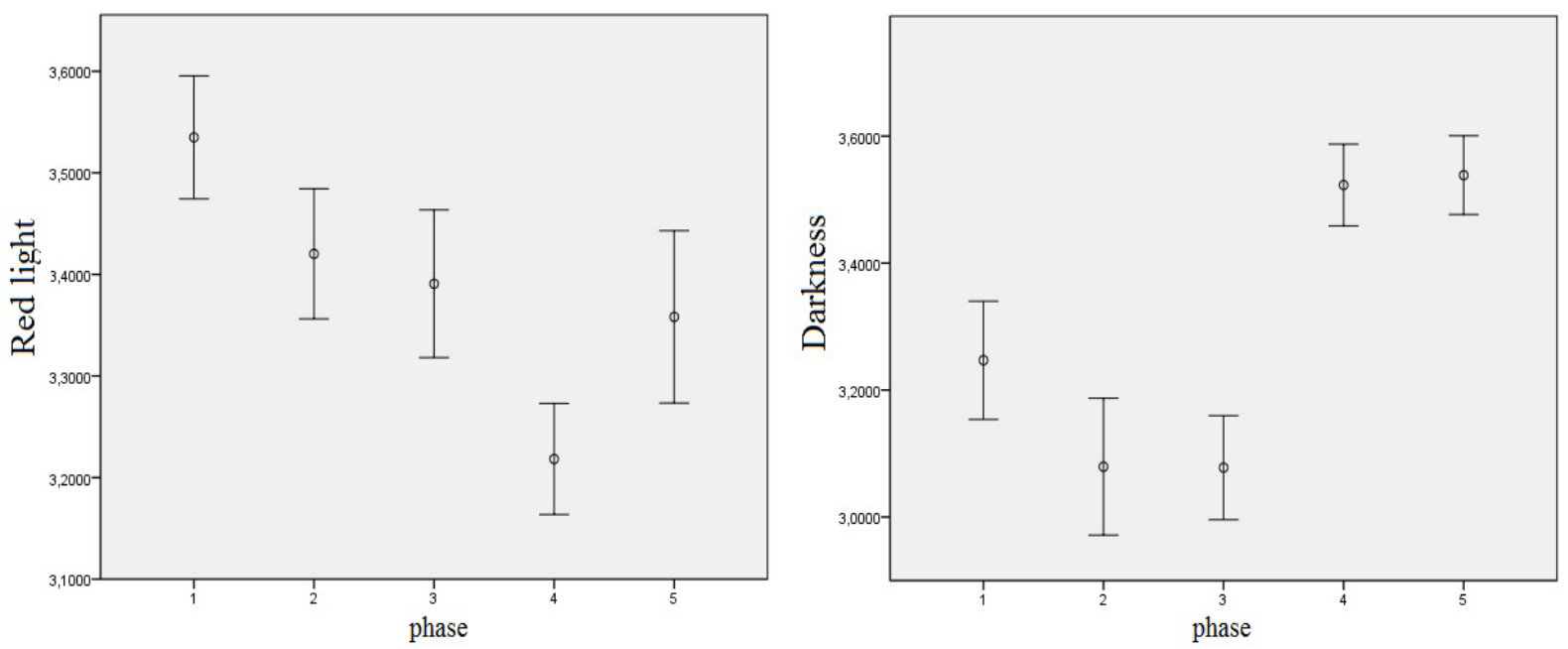

FIGURE 6.

Means of log-transformed alpha EEG power density in the RL and in the DC in different phases with error bars representing \pm 1 standard error, regardless of derivation.

\section{TABLE 8.}

The Three-Factorial ANOVA Results for the Alpha Frequencies

\begin{tabular}{ccc}
\hline Phase & Phase & $p$ \\
\hline 1 & 2 & .041 \\
\hline \multirow{2}{*}{2} & 4 & .024 \\
\cline { 2 - 3 } & 5 & .049 \\
\hline \multirow{2}{*}{3} & 4 & 0.24 \\
\cline { 2 - 3 } & 5 & 0.49 \\
\hline
\end{tabular}

\section{TABLE 9.}

Oz Derivation: Significant Test Results of the Pairwise Comparisons with the Bonferroni Correction for the Interaction of the Light and Phase

\begin{tabular}{cccc}
\hline Light condition & Phase & Phase & $p$ \\
\hline Blue light & 3 & 4 & .01 \\
Red light & 1 & 4 & .034 \\
& 3 & 4 & .047 \\
\hline
\end{tabular}

With regard to the $\mathrm{Oz}$ derivation, the interaction between light and measurement phase was significant as well, $F(8,144)=4.079, p<.001$. Further analysis showed that significant differences were noticeable for BL and RL, as shown in Table 9 and Figure 8. In both RL and BL conditions, mean alpha frequencies were significantly higher in Phase 3 than in Phase 4 in the $\mathrm{Oz}$ derivation. Such a decrease also concerned RL between Phases 1 and 4 .

\section{The Karolinska Sleepiness Scale}

In order to compare the baseline levels of alertness ratings and cognitive performance pre-exposure, the appropriate tests (Student's $t$ and Wilcoxon signed rank tests, depending on the assumption fulfillment) were conducted. All significances exceeded the .05 threshold, which suggests that the participants' levels of alertness did not significantly differ over the three days of the experiment.

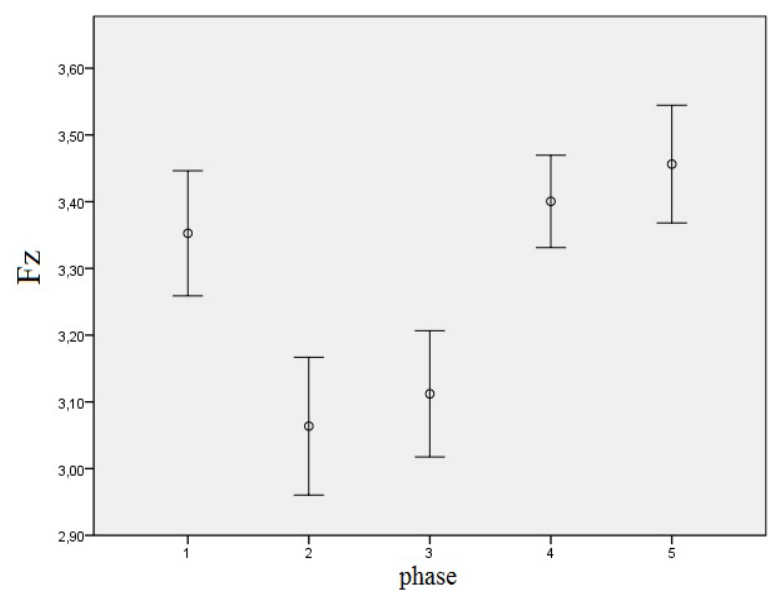

FIGURE 7.

Mean log-transformed alpha EEG power density in the Fz derivation and different phases with error bars representing $\pm 1 \mathrm{SE}$, regardless of light condition.

We were curious about the KSS results and their comparison before and after exposure to the various light conditions. At the beginning, of primary interest were three variables describing differences in KSS results before and after BL, RL, and the DC. The Wilcoxon signed-rank test for BL and the DC, together with the Student's $t$ test for two dependent samples related to RL were performed in order to compare the results before and after light exposure. The results concerning BL $(T=128.5 ; p=.164)$ and $\mathrm{RL}(t[18]=-1.455, p=.163)$ do not allow us to reject the hypothesis that there are no differences between the medians/means of the KSS results before and after exposures. However, in the case of the DC, the Wilcoxon signed-rank test proved to be significant $(T=114, p=.002)$, which implies that there was a difference between the medians of the KSS results before and after the DC. In particular, the KSS scores after the DC increased compared to the beginning conditions of the experiment (see Figure 9). 


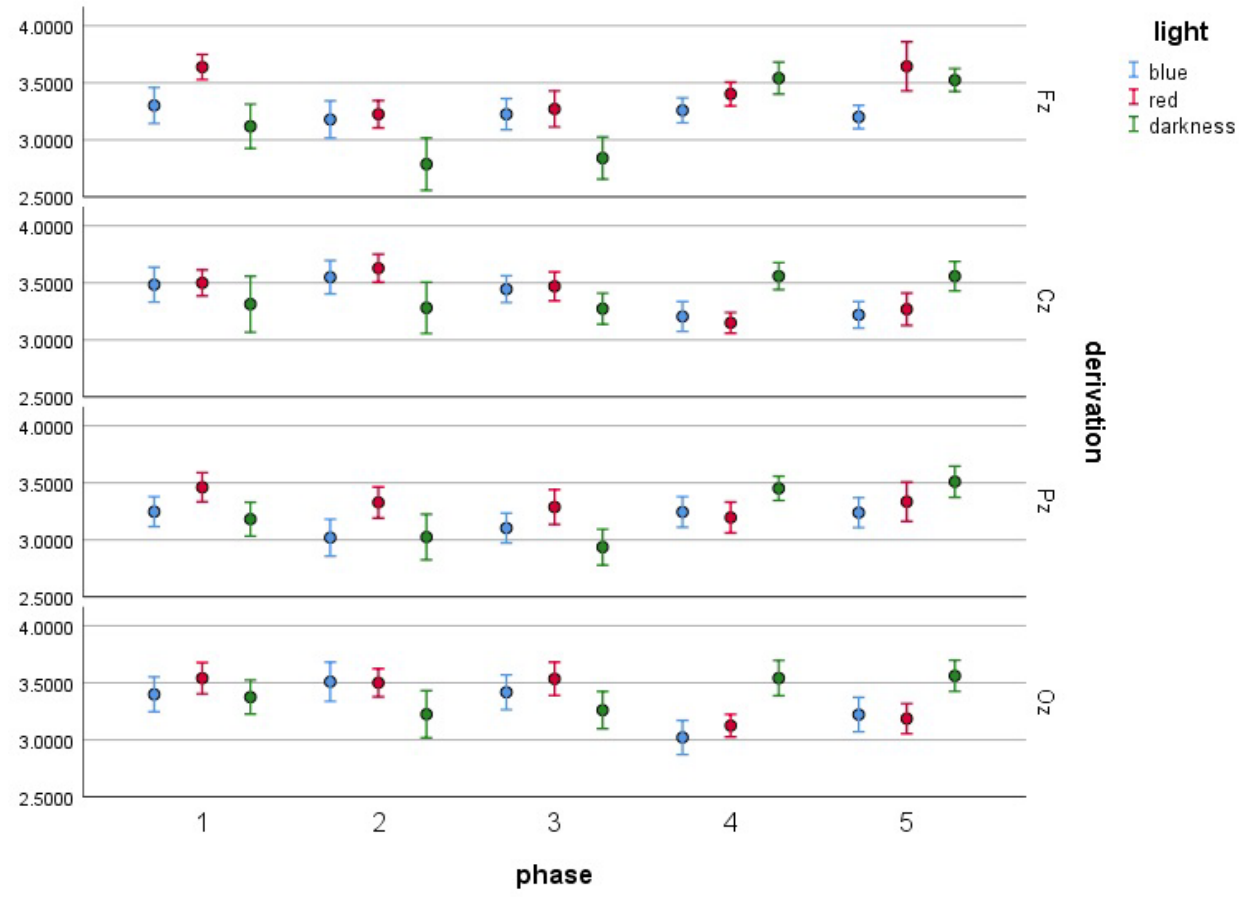

FIGURE 8.

Means of log-transformed alpha EEG power density in various derivations ( $F z, C Z, P z, O z)$ and light conditions with error bars representing $\pm 1 \mathrm{SE}$.

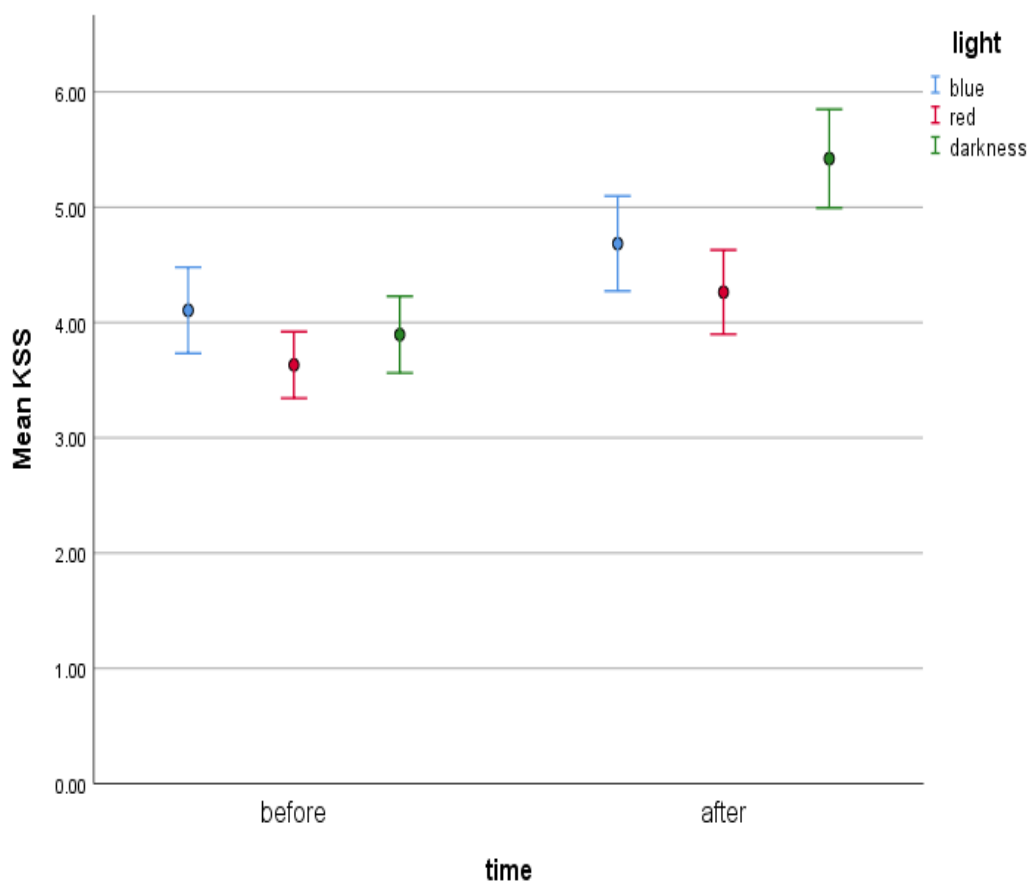

FIGURE 9.

Means of KSS scores in various light conditions before and after light exposure with error bars representing \pm 1 SE. 


\section{Performance}

First, we explored the relations between the subjective measures of alertness and cognitive performance by conducting correlation tests along with calculating Pearson's correlation coefficients. In most cases, these measures proved to not be significantly correlated, except for the difference in the KSS scores before and after BL and the difference in time of incorrect $X$ reactions in the CPT II before and after BL $(r=0.476, p=.04)$, the KSS scores before BL and the amount of incorrect $X$ reactions in the CPT II before $\mathrm{BL}(r=0.514, p=.024)$, as well as between the amount of correct $X$ after $A$ reactions in the CPT II before $\mathrm{BL}(r=0.576, p=.01)$, the KSS scores after the BL and SRT reaction time after BL $(r=0.629, p=.004)$, and the KSS scores after the DC and the amount of correct other reactions in the CPT II after the DC $(r=-0.465, p=.045)$.

We examined three cognitive tests comparing the participants' efficiency in various lighting conditions. Nowhere did we find any significant differences except for one of the factors in the CPT II: the time of the correct $X$ after $A$ reactions before and after the RL exposure.

In order to ascertain whether dissimilarities between the CPT II time of the correct $X$ after $A$ reactions before and after the light exposure in various lighting conditions occur, we examined the differences of the respective variables for $\mathrm{BL}, \mathrm{RL}$, and the $\mathrm{DC}$.

The results show that the differences between the mean CPT II time of the correct $X$ after $A$ reactions before and after the BL exposure are not significant, $t(18)=1.164, p=.26$. The results were insignificant for the DC also, $z=-0.04, p=.984$. Unexpectedly, the results for RL showed significant differences before and after the exposure, $z=-2.133, p=.016$. The results indicate that the CPT-II correct re- sponse time before the RL exposure $(M d n=504,68)$ was significantly lower than after $(M d n=533.68)$. The respective box plots illustrating the medians are presented in Figure 10.

\section{DISCUSSION}

The goal of the current study was to compare the influence of BL (465 $\mathrm{nm})$ with $\mathrm{RL}(625 \mathrm{~nm})$ on objective and subjective measures of alertness during the day so as to extend the results of Figueiro et al. (2009), Sahin and Figueiro (2013; Sahin et al., 2014), and Łaszewska et al. (2017). Investigating the brightest part of the $24 \mathrm{~h}$ day-noon (Klejna et al., 2014) - we introduced the next part of the lighting scheme. We were interested in whether the NVAR is induced by a nonvisual system other than the circadian one. Correspondingly, with this research, we offer some new information about the acute NVAR to RL. With regard to the EEG data, we found that exposure to light (both RL and BL) did not significantly affect power density in the theta waves compared to the DC, where we observed an increase over time. Exposure to RL at noon significantly reduced alpha power over time compared to when subjects remained in the DC; it did so; however, in small range. Exposure to BL did not affect alpha power. The results are consistent only with the hypothesis that RL enhances alertness measures in the EEG. They are contrary to our hypothesis that both BL and RL, unlike the DC, would enhance the alertness level observed by the decrease in the EEG power density of the theta and alpha ranges.

We hypothesized that BL and RL, unlike the DC, would enhance the alertness level, as observed by the improvement of reaction time and the number of correct reactions in the cognitive tests. It turned

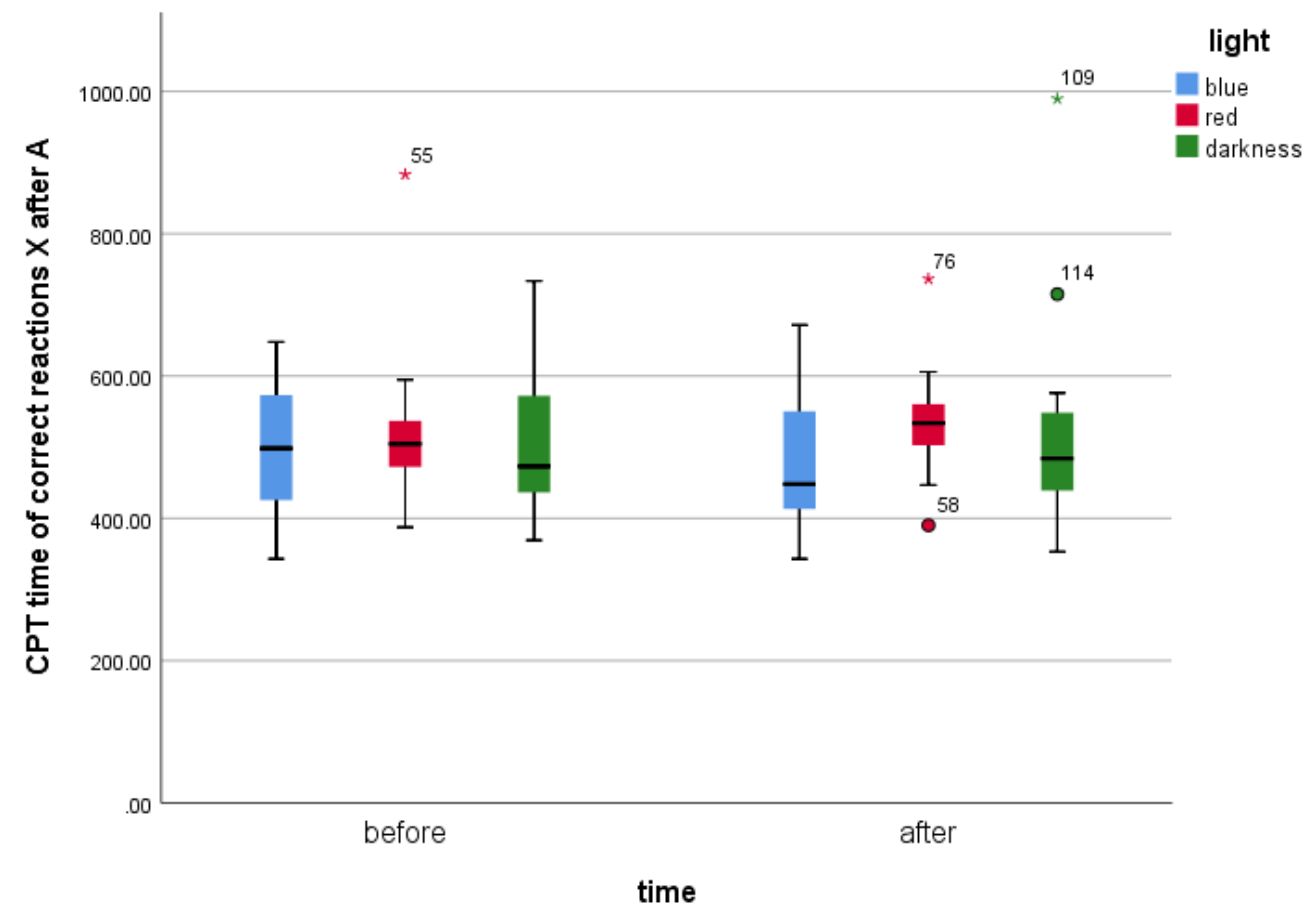

FIGURE 10.

Medians of CPT-II time of correct X after A reactions in various light conditions before and after the light exposure. 
out that all the differences examined were not significant, apart from the one concerning RL exposure in the CPT II in one of its six factors, the time of correct responses. Surprisingly, participants performed significantly slower after exposure to RL. The results were contrary to this assumption since $\mathrm{BL}$ did not translate into better performance in any of the applied tests.

We also hypothesized that both BL and RL, unlike the DC, would enhance the alertness level observed by the decrease of subjective sleepiness, as measured by the KSS. Subjective measures of alertness were not affected by light in either of the conditions, in contrast to the DC, where the subjects reported greater sleepiness than before. In particular, the KSS scores after the DC increased when compared to the beginning conditions of the experiment.

The results observed are contrary to what we assumed, which was that light is a robust countermeasure for subjective sleepiness and for performance, lessening the growing sleepiness in humans at noon. On the basis of these few EEG effects, we conclude that sleepiness was not reduced by light at noon, but was rather induced by darkness, since high EEG power density in the theta ranges has been associated with sleepiness. These effects contradict what was shown in previous findings where, in the afternoon, exposure to RL significantly decreased EEG power density in the theta, alpha theta (Sahin \& Figueiro, 2013), and alpha ranges (Sahin \& Figueiro, 2013; Sahin et al., 2014). These differences may have occurred because light exposures in the present study occurred $5 \mathrm{~h}$ after habitual wake times while in the studies of Sahin and Figueiro (2013), light exposures occurred $7 \mathrm{~h}$ after wake times. Thus, homeostatic pressure might have triggered greater response on light stimulation (Phipps-Nelson, Redman, Dijk, \& Rajaratnam, 2003; Rüger, Gordijn, Beersma, de Vries, \& Daan, 2006). However, this does not completely account for the differences. The results of the current study are also inconsistent with very recent findings from measurements taken at the same time of day, where decreases in synchronization in upper theta, lower alpha, and upper alpha EEG activity were observed (Łaszewska et. al, 2017). We observed that BL did not induce the NVAR, unlike what has been well documented during the nighttime (Ben-Shlomo \& Kyriacou, 2010; Cajochen et al., 2000; Cajochen, Wyatt, Czeisler, \& Dijk, 2002; Cajochen et al., 2005; Chellappa et al., 2011; Chellappa et al., 2013; Figueiro, Bullough, Bierman, Fay, \& Rea, 2007; Figueiro et al., 2009; Lockley et al., 2003; Lockley et al., 2006; Plitnick et al., 2010; Zeitzer, Dijk, Kronauer, Brown, \& Czeisler, 2000) and during the daytime, where a significant decrease in the EEG power density of alpha ranges after exposure to white light containing $\mathrm{BL}$ radiation were observed (Sahin et al., 2014). An explanation for these daytime differences may be the influence of other parts of the light spectrum than BL present in white light, whereas in the current study, only monochromatic lights were examined. However, this is just a preliminary assumption which demands further study. The results obtained contradict the functional magnetic resonance imaging (fMRI) study of Vandewalle et al. (2006), which showed a greater NVAR after exposure to blue radiation $(480 \mathrm{~nm})$ than when exposed to shorter $(430 \mathrm{~nm})$ or longer $(530 \mathrm{~nm})$ radiation. A possible explanation of these differences may be that the fMRI used in their study might have been more sensitive to brain activity changes than the EEG power density. Moreover, it should be noted that they did not examine the impact of $\mathrm{RL}(630 \mathrm{~nm})$ as we did. When it comes to alpha waves, we concluded that during the daytime, the alpha range might not be equally sensitive to every light stimulus. It might be more sensitive to RL stimuli than to BL stimuli. However, this conclusion needs further study.

Since the effects of light exposure on performance are inconsistent (Souman et. al, 2017), we decided to observe cognitive processes beyond simple reaction time. In our study, the effects of light on cognitive-behavioral performance obtained in our study were sparse. Despite the number of tests performed, we obtained only one significant result. This is contrary to what was shown during the daytime in the fMRI studies of Vandewalle, Liang, et al. (2006) and Vandewalle et al. (2007), which aimed at demonstrating that BL almost instantaneously influences activity in brain areas involved in working memory and in the modulation of cognition by arousal. However, our results are consistent with the results obtained during the nighttime, when no changes in reaction time were observed (Figueiro et al., 2009). When it comes to RL, the effects were not consistent with those from the afternoon, where short-term performance worsened after exposure to RL (Sahin et al., 2014). We, however, did not observe any effects. One potential explanation for the detrimental influence of RL on performance might relate to the effect of color. Red color is suspected to increase human receptiveness to external stimuli and increase excitation, and thus, affect emotional and motor responses (Kwallek, Soon, \& Lewis, 2007). It seems that light seen as red at noon rather impedes the sustaining of attention, executive functions, and cognitive efficiency. However, we cannot refer to this explanation, since color is rather the psychological result of processing by neural mechanisms of the spectral irradiance distribution (SID) incident on the retina, not a stimulus, as light is. This deterioration in performance is consistent with the spontaneously recorded statements of the participants after the experiment sessions. They reported emotional arousal after the RL condition. However, their subjective experiences were not controlled in the study procedure, so we cannot draw any conclusions on this basis. We consider this a limitation of the present paper and suggest including emotion assessment in future studies. Another possible explanation for the sparse effect of light stimuli is the low level of light. Some studies have shown that higher levels of light (1000 lux) improved performance during the daytime, while no effect was observed at lower levels of light (200 lux, Phipps-Nelson et al., 2003; Rüger et al., 2006; Smolders, de Kort, \& Cluitmans, 2012). In contrast, Badia, Myers, Boecker, Culpepper, and Harsh (1991) showed that 2500 lux of white light increased performance during the nighttime, but not during the daytime. These conflicting results might be partly explained by the fact that the effects obtained in the studies of Phipps-Nelson et al. (2003) and Rüger et al. (2006) were amplified through sleep deprivation, whereas those of Vandewalle et al. $(2006 ; 2007)$ were observed instantaneously while conducting tasks. We cannot explain our results by referencing the low level of illumination, since the results are inconsistent. Ultimately, we want to emphasize that performance is difficult to measure and that boredom might play some role in these sparse effects, which subjects 
informally reported after sitting in front of a light box for nearly an hour. Although RL triggered some deterioration in performance, these results are poor and demand further study. Additional studies should examine if the cognitive-behavioral effect exists at all and how light, especially RL, can modulate the different types of performance at daytime.

The results of the subjective alertness measurement did not support our hypothesis that light is a robust countermeasure for subjective sleepiness, as we did not observe any alerting effect of light, but rather sleepiness in the dim light. One possible explanation for these results is that rather than reflecting a subject's subjective sleepiness, the KSS scores may have been reflecting the boredom that they reported after sitting in front of a light box for nearly one hour. The present results contradict the study of Phipps-Nelson et al. (2003), where a $5 \mathrm{~h}$ exposure to bright light at 1000 lux reduced subjective sleepiness when compared to dim light. In our opinion, for both BL and RL to trigger subjective alertness at noon, it may be necessary to have longer exposure durations or higher levels of light than only 40 lux. Moreover, the changes in the EEG power density triggered by RL exposure were not experienced by the participants at noon. However, this may be due to very sparse changes in brain activity. Furthermore, self-reported measures often correlate poorly with objective measures of alertness (Zhou et al., 2012). The Phipps-Nelson study together with studies by Åkerstedt and Gillberg (1990) and Waage et al. (2012) indicate that the association between the KSS and objective measures of sleepiness is not easily seen when sleep pressure is low. Therefore, we suggest that future studies investigate whether the correlation between KSS scores and objective measures of alertness at noon is greater when subjects are sleep deprived. Lastly, we would like to stress that the limitation of self-reported measures is such that it is difficult to keep subjects blind to light manipulations, so their responses may be influenced by their expectations or associations.

We conclude that our findings may add to the growing body of evidence that at noon, behavioral effects of light might be very limited. It must be taken into consideration, however, that we only obtained several effects and that they were inconsistent; therefore, we consider our conclusion just a preliminary assumption in need of additional studies. It should be noted that the current results do not rule out the possibility that RL and BL trigger the NVAR at noon. Perhaps research with a larger sample size or with light levels higher than 40 lux would lead to statistically significant changes in EEG power density in the theta and alpha (power) ranges, in performance and in subjective alertness. Lastly, it is important to observe how the entire spectrum of emitted light, not only $\mathrm{BL}$, affects human physiology during the daytime.

\section{ACKNOWLEDGEMENTS}

The equipment for the study was sponsored by Nicolaus Copernicus University Ludwik Rydygier Collegium Medicum in Bydgoszcz. This research did not receive any specific grant from funding agencies in the public, commercial, or not-for-profit sectors. We are grateful to Dr Mariana Figueiro for sharing her experience. We thank the subject volunteers for their help

\section{REFERENCES}

Arendt, J., Bojkowski, C., Franey, C., Wright, J., \& Marks, V. (1985). Immunoassay of 6-hydroxymelatonin sulfate in human plasma and urine: abolition of the urinary 24-hour rhythm with atenolol. The Journal of Clinical Endocrinology \& Metabolism, 60, 1166-1173. doi: 10.1210/jcem-60-6-1166. [WwW|

Arendt, J., Deacon, S., English, J., Hampton, S., \& Morgan, L. (1995). Melatonin and adjustment to phase shift. Journal of Sleep Research, 4, 74-79. doi: 10.1111/j.1365-2869.1995.tb00232.x wwW

Åkerstedt, T., \& Gillberg, M. (1990). Subjective and objective sleepiness in the active individual. International Journal of Neuroscience, 52, 29-37. doi: 10.3109/00207459008994241 WWW

Baddeley, A. D., \& Logie, R. H. (1999). Working memory: the multiple-component model. In A. Miyake, P. Shah (Eds.), Models of working memory (pp. 28-61). New York City, NY: Cambridge University Press.

Badia, P., Myers, B., Boecker, M., Culpepper, J., \& Harsh, J. R. (1991). Bright light effects on body temperature, alertness, EEG and behavior. Physiology \& Behavior, 50, 583-588. doi: 10.1016/0031-9384(91)90549-4 [www

Beck, A. T., Steer, R. A., Carbin, M. G. (1988). Psychometric properties of the Beck Depression Inventory: Twenty-five years of evaluation. Clinical Psychology Review, 8, 77-100. doi: 10.1016/0272-7358(88)90050-5 [WW|

Ben-Shlomo, R., \& Kyriacou, C. P. (2010). Light pulses administered during the circadian dark phase alter expression of cell cycle associated transcripts in mouse brain. Cancer Genetics and Cytogenetics, 197, 65-70. doi: 10.1016/j.cancergencyto.2009.11.003 WWW

Berson, D. M., Dunn, F. A., \& Takao, M. (2002, February). Phototransduction by retinal ganglion cells that set the circadian clock. Science, 295, 1070-1073. doi: 10.1126/science.1067262 [WWW

Brainard, G., Hanifin, J., Greeson, J., Byrne, B., Glickman, G., ..., Rollag, M. D. (2001). Action spectrum for melatonin regulation in humans: Evidence for a novel circadian photoreceptor. Journal of Neuroscience, 21, 6405-6412. doi: 10.1523/ JNEUROSCI.21-16-06405.2001 www

Buysse, D. J., Reynolds, C. F., Monk, T. H., Berman, S. R., \& Kupfer, D. J. (1989). The Pittsburgh Sleep Quality Index: A new instrument for psychiatric practice and research. Psychiatry Research, 28, 193-213. doi: 10.1016/S0960-9822(02)00659-0 www

Cahill, L. (2006). Why sex matters for neuroscience. Nature Reviews Neuroscience, 7, 477-484. doi: 10.1038/nrn1909 [WWW

Cajochen, C., Frey, S., Anders, D., Späti, J., Bues, M., ... Stefani, O. (2011). Evening exposure to a light-emitting diodes (LED)backlit computer screen affects circadian physiology and cognitive performance. Journal of Applied Physiology, 110, 1432-1438. doi: 10.1152/japplphysiol.00165.2011 www

Cajochen, C., Munch, M., Kobialka, S., Krauchi, K., Steiner, R., ... Wirz-Justice, A. (2005). High sensitivity of human melatonin, alertness, thermoregulation and heart rate to short wave- 
length light. The Journal of Clinical Endocrinology \& Metabolism, 90, 1311-1316. doi: 10.1210/jc.2004-0957 |WwW

Cajochen, C., Wyatt, J. K., Czeisler, C. A., \& Dijk, D. J. (2002). Separation of circadian and wake duration-dependent modulation of EEG activation during wakefulness. Neuroscience, 114, 1047-1060. doi: 10.1016/\$0306-4522(02)00209-9 Www

Cajochen, C., Zeitzer, J. M., Czeisler, C. A., \& Dijk, D. J. (2000). Dose-response relationship for light intensity and ocular and electroencephalographic correlates of human alertness. Behavioural Brain Research, 115, 75-83. doi: 10.1016/S01664328(00)00236-9

Chellappa, S. L., Steiner, R., Blattner, P., Oelhafen, P., Götz, T., \& Cajochen, C. (2011). Non-visual effects of light on melatonin, alertness and cognitive performance: can blue-enriched light keep us alert? PLoS One, 6, e16429. doi: 10.1371/journal. pone.0016429 WwW

Chellappa, S. L., Steiner, R., Oelhafen, P., Lang, D., Götz, T., ... Cajochen, C. (2013). Acute exposure to evening blue-enriched light impacts on human sleep. Journal of Sleep Research, 22, 573-580. doi: 10.1111/jsr.12050 Www

Crowley, S. J., Lee. C., Tseng, CY., Fogg, L, F., \& Eastman C. I. (2003). Combinations of bright light, scheduled dark, sunglasses, and melatonin to facilitate circadian entrainment to night shift work. Journal of Biological Rhythms, 18, 513-523. doi: $10.1177 / 0748730403258422$ [www]

Czeisler, C. A. (2009). Medical and genetic differences in the adverse impact of sleep loss on performance: ethical considerations for the medical profession. Transactions of the American Clinical and Climatological Association, 120, 249-285. WWW

Czeisler, C. A., \& Gooley, J. J. (2007). Sleep and circadian rhythms in humans. Cold Spring Harbor Symposia on Quantitative Biology, 72, 579-97. doi: 10.1101/sqb.2007.72.064 |wWW|

Dijk D-J, Archer S.N. (2009). Light, sleep, and circadian rhythms: Together Again. PLoS Biology, 7(6): e1000145. doi: 10.1371/ journal.pbio.1000145 WWW|

Eastman, C. I., Stewart, K. T., Mahoney, M. P., Liu, L., \& Fogg, L. F. (1994). Dark goggles and bright light improve circadian rhythm adaptation to night-shift work. Sleep, 17, 535-543. doi: 10.1093/sleep/17.6.535

Figueiro, M. G., Bullough, J. D., Bierman, A., Fay, C. R., \& Rea, M. S. (2007). On light as an alerting stimulus at night. Acta Neurobiologiae Experimentalis, 67, 171-178. [WWW]

Figueiro, M. G., Bierman, A., Plitnick, B., \& Rea, M. S. (2009). Preliminary evidence that both blue and red light can induce alertness at night. BMC Neuroscience, 10, 105. doi: 10.1186/1471-2202-10-105 [WwW

Fonken, L. K., Finy, M. S., Walton, J. C., Weil, Z. M., Workman, J. L., ... Nelson, R. J. (2009). Influence of light at night on murine anxiety- and depressive-like responses. Behavioural Brain Research, 205, 349-54. doi: 10.1016/j.bbr.2009.07.001 [WWW

Hall , D. M. B., \& Cole, T. J. (2006). What use is the BMI? Archives of Disease in Childhood, 9, 283-286. doi: 10.1136/adc.2005.077339 www
Horne, J. A., \& Ostberg, O. (1976). A self-assessment questionnaire to determine morningness-eveningness in human circadian rhythms. International Journal of Chronobiology, 4, 97-110. |Ww| Jain, A., Bansal, R., Kumar, A., \& Singh, K. D. (2015). A comparative study of visual and auditory reaction times on the basis of gender and physical activity levels of medical first year students. International Journal of Applied and Basic Medical Research, 5, 124-127. doi: 10.4103/2229-516X.157168 WWW

Jansma, J. M., Ramsey, N. F., Coppola, R., \& Kahn, R. S. (2000). Specific versus nonspecific brain activity in a parametric $\mathrm{N}$-back task. Neuroimage, 12, 688-697. doi: 10.1006/nimg.2000.0645 WWW

Johns, M. W. (1991). A new method for measuring daytime sleepiness: The Epworth sleepiness scale. Sleep, 14, 540-545. doi: 10.1093/sleep/14.6.540 WwW

Kaida, K., Takahashi, M., Akerstedt, T., Nakata, A., Otsuka, Y., ... Fukasawa, K. (2006). Validation of the Karolinska sleepiness scale against performance and EEG variables. Clinical Neurophysiology, 117, 1574-1581. doi: 10.1016/j. clinph.2006.03.011 wWw

Klejna, M., Uscka-Kowalkowska, J., Araźny, A., Kunz, M., Maszewski, R., \& Przybylak, R. (2014). Spatial differentiation of global solar radiation in Torun and its suburban area (Central Poland) in 2012. Bulletin of Geography - Physical Geography Series, 7, 27-54. doi: 10.2478/bgeo-2014-0002 [WwW

Klerman, E. B., Shanahan, T. L., Brotman, D. J., Rimmer, D. W., Emens, J. S., ... Czeisler, C. A. (2002). Photic resetting of the human circadian pacemaker in the absence of conscious vision. Journal of Biological Rhythms, 17, 548-555. doi: $10.1177 / 0748730402238237$ WwW

Kwallek, N., Soon, K., \& Lewis, C. (2007). Work week productivity, visual complexity, and individual environmental sensitivity in three offices of different color interiors. Color Research and Application, 32, 130-143. doi: 10.1002/col.20298 wWw

Lakowski, R. (1969). Theory and practice of colour vision testing: A review. British Journal of Industrial Medicine, 26, 265-288. WWW

Lewy, A., Wehr, T., Goodwin, T., Newsome, D., \& Markey, S. (1980, December). Light suppresses melatonin secretion in humans. Science, 210, 1267-1269. doi: 10.1126/science.7434030 www

Lockley, S. W., Brainard G. C., \& Czeisler, C. A. (2003). High sensitivity of the human circadian melatonin rhythm to resetting by short wavelength light. The Journal of Clinical Endocrinology \& Metabolism, 88, 4502-4505. doi: 10.1210/jc.2003-030570 [wWW

Lockley, S. W., Evans, E. E., Scheer, F., Brainard, G. C., Czeisler C. A., \& Aeschbach, D. (2006). Short-wavelength sensitivity for the direct effects of light on alertness, vigilance, and the waking electroencephalogram in humans. Sleep, 29, 161-168. doi: 10.1093/sleep/29.2.161 |wWw

Lockley, S. W., \& Gooley, J. J. (2006). Circadian photoreception: Spotlight on the brain. Current Biology, 16, 795-797. doi: 10.1016/j.cub.2006.08.039 WWW

Lucas, R. J., Peirson, S. N., Berson, D. M., Brown, T. M., Cooper, H. M., ... Brainard, G. C. (2014). Measuring and using light 
in the melanopsin age. Trends in Neurosciences, 37, 1-9. doi: 10.1016/j.tins.2013.10.004 WWW

Łaszewska, K., Goroncy, A., Weber, P., Pracki, T., Tafil-Klawe, M., ... Złomańczuk, P. (2017). Daytime acute non-visual alerting response in brain activity occurs as a result of short- and longwavelengths of light. Journal of Psychophysiology. Advance online publication. doi: 10.1027/0269-8803/a000199 |wWW

Martin, S. K., \& Eastman, C. I. (1997). Medium-intensity light produces circadian rhythm adaptation to simulated night-shift work. Sleep, 21, 154-165. doi: 10.1093/sleep/21.2.154 |WWW

Maycock, G. (1996). Sleepiness and driving: the experience of UK car drivers. Journal of Sleep Research, 5, 229-237. doi: 10.1111/j.1365-2869.1996.00229.x WWW

Mills, P. R., Tomkins, S. C., \& Schlangen, L. J. M. (2007). The effect of high correlated colour temperature office lighting on employee wellbeing and work performance. Journal of Circadian

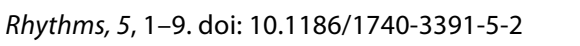

Monk, T. H. (2005). The post-lunch dip in performance. Clinics in Sports Medicine, 24, e15-23. doi: 10.1016/j.csm.2004.12.002 WWW

Oken, B. S., Salinsky, M. C., \& Elsas, S. M. (2006). Vigilance, alertness, or sustained attention: physiological basis and measurement. Clinical Neurophysiology, 117, 1885-1901. doi: 10.1016/j. clinph.2006.01.017 WWW

Penev, P. D., Kolker, D. E., Zee, P. C., \& Turek, F. W. (1998). Chronic circadian desynchronization decreases the survival of animals with cardiomyopathic heart disease. American Journal of Physiology, 275, H2334-7. doi: 10.1152/ ajpheart.1998.275.6.H2334 |WWW

Phipps-Nelson, J., Redman, J. R., Dijk, D. J., \& Rajaratnam, S. M. (2003). Daytime exposure to bright light, as compared to dim light, decreases sleepiness and improves psychomotor vigilance performance. Sleep, 26, 695-700. doi: 10.1093/ sleep/26.6.695 wWw

Plitnick, B., Figueiro, M. G., Wood, B., \& Rea, M. S. (2010). The effects of red and blue light on alertness and mood at night. Lighting Research \& Technology, 42, 449-458. doi: $10.1177 / 1477153509360887$ WWW

Rea, M. S., Figueiro, M. G., Bullough, J. D., \& Bierman, A. (2005). A model of phototransduction by the human circadian system. Brain Research Reviews, 50, 213-228. doi: 10.1016/j.brainres-

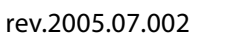

Revell, V. L., \& Eastman, C. I. (2005). How to trick mother nature into letting you fly around or stay up all night. Journal of Biological Rhythms, 20, 353-365. doi: 10.1177/0748730405277233 |

Reyner, L. A., \& Horne, J. A. (2000). Early morning driver sleepiness: Effectiveness of $200 \mathrm{mg}$ caffeine. Psychophysiology, 37, 251-256. WWW

The Royal Society for the Prevention of Accidents (2008). Driver fatigue and road accidents factsheet. Retrieved from http:// www.rospa.com/rospaweb/docs/advice-services/road-safety/ drivers/driver-fatigue-2011.pdf

The Royal Society for the Prevention of Accidents. (2011). Driver fatigue and road accidents. Retrieved from http://www.rospa. com/road-safety/advice/drivers/fatigue/road-accidents

Rüger, M., Gordijn, M. C., Beersma, D. G., de Vries, B., \& Daan, S. (2006). Time-of-day-dependent effects of bright light exposure on human psychophysiology: comparison of daytime and nighttime exposure. American Journal of PhysiologyRegulatory, Integrative, and Comparative Physiology, 36, 148-151. doi: 10.1152/ajpregu.00121.2005 WWW

Sahin, L., \& Figueiro, M. G. (2013). Alerting effects of short-wavelength (blue) and long-wavelength (red) lights in the afternoon. Physiology \& Behavior, 116, 1-7. doi: 10.1016/j.physbeh $\underline{\underline{W W}}$

Sahin, L., Wood, B. M., Plitnick, B., \& Figueiro, M. G. (2014). Daytime light exposure effects on biomarkers, measures of alertness, and performance. Behavioural Brain Research, 274, 176-185. doi: 10.1016/j.bbr.2014.08.017 |WWW

Scheer, F., \& Buijs, R. M. (1999). Light affects morning salivary cortisol in humans. The Journal of Clinical Endocrinology and Metabolism, 84, 3395-3398. doi: 10.1210/jcem.84.9.6102 WWW

Schernhammer, E. S., \& Schulmeister K. (2004). Melatonin and cancer risk: Does light at night compromise physiologic cancer protection by lowering serum melatonin levels? British Journal of Cancer, 90, 941-943. WWW

Smith, M. R., \& Eastman, C. I. (2008). Night shift performance is improved by a compromise circadian phase position: Study 3. Circadian phase after 7 night shifts with an intervening weekend off. Sleep, 31, 1639-1645. doi: 10.1093/sleep/31.12.1639 WWW Smolders, K. C., de Kort, Y. A., \& Cluitmans, P. J. (2012). A higher illuminance induces alertness even during office hours: findings on subjective measures, task performance and heart rate measures. Physiology \& Behavior, 107, 7-16. doi: 10.1016/j. physbeh.2012.04.028

Souman, J. L., Tinga, A., Te Pas, S. F., Van Ee, R., \& Vlaskamp, B. (2017). Acute alerting effects of light: A systematic literature review. Behavioral Brain Research, 337, 228-239. doi: 10.1016/j. bbr.2017.09.016 $\overline{\mathrm{WWW}}$

Terman, M., \& Williams, J. B. W. (2015). Personal inventory for depression and seasonal affective disorder (PIDS). doi: 10.13140/

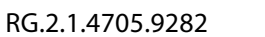

Vandewalle, G., Balteau, E., Phillips, C., Degueldre, C., Moreau, V., ... Dijk, D. J. (2006). Daytime light exposure dynamically enhances brain responses. Current Biology, 16, 1616-1621. doi: 10.1016/j.cub.2006.06.031 $\underline{\underline{W W}}$

Vandewalle, G., Liang, X., Jacobs, R., \& Lambrichts, I. (2006). Macroanatomic and radiologic characteristics of the superior genial spinal foramen and its bony canal. The International Journal of Oral \& Maxillofacial Implants, 21, 581-586.

Vandewalle, G., Maquet, P., \& Dijk, D. J. (2009). Light as a modulator of cognitive brain function. Trends in Cognitive Sciences, 13, 429-438. doi: 10.1016/j.tics.2009.07.004 WWW

Vandewalle, G., Schmidt, C., Albouy, G., Sterpenich, V., Darsaud, A., ... Dijk, D. J. (2007). Brain responses to violet, blue, and green monochromatic light exposures in humans: promi- 
nent role of blue light and the brainstem. PLoS One, 2, e1247. doi: 10.1371/journal.pone.0001247 WwW

Vandewalle, G., Schwartz, S., Grandjean, D., Wuillaume, C., Balteau, E., ... Maquet, P. (2010). Spectral quality of light modulates emotional brain responses in humans. Proceedings of the National Academy of Sciences of the United States of America, 107, 19549-19554. doi: 10.1073/pnas.1010180107 WWW

Vanlaar, W. (2008). Less is more: The influence of traffic count on drinking and driving behaviour. Accident Analysis \& Prevention, 40, 1018-1022. doi: 10.1016/j.aap.2007.11.007 WwW

Wong, K. Y., Dunn, F. A., \& Berson, D. M. (2005). Photoreceptor adaptation in intrinsically photosensitive retinal ganglion cells. Neuron, 48, 1001-1010. doi: 10.1016/j.neuron.2005.11.016 [WWW

Waage, S., Harris, A., Pallesen, S., Saksvik, I. B., Moen, B. E., \& Bjorvatn, B. (2012). Subjective and objective sleepiness among oil rig workers during three different sleep schedules. Sleep Medicine, 13, 64-72. doi: 10.1016/j.sleep.2011.04.009 [wW]
Zeitzer, J. M., Dijk, D. J., Kronauer, R. E., Brown, E. N., \& Czeisler, C. A. (2000). Sensitivity of the human circadian pacemaker to nocturnal light: melatonin phase resetting and suppression. The Journal of Physiology, 526, 695-702. doi: 10.1111/j.14697793.2000.00695.x [www]

Zhou, X., Ferguson, S. A., Matthews, R. W., Sargent, C., Darwent, D., ... Roach, G. D. (2012). Mismatch between subjective alertness and objective performance under sleep restriction is greatest during the biological night. Journal of Sleep Research, 21,

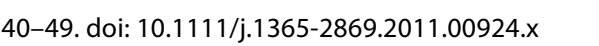

Zyss, T., Hesse, R. T., \& Zięba, A. (2009). Rozmieszczanie elektrod podstawowych w układzie 10-20 [Arranging primary electrodes in a 10-20 system]. In T. Zyss (Ed.), Zastosowanie układu 10-20 w rozmieszczaniu elektrod do EEG [Application of the 1020 system in the placement of electrodes to EEG] (pp. 36-62). Warsaw, Poland: Biblioteka Elmiko.

RECEIVED 11.10.2018 | ACCEPTED 26.11.2018 